\title{
Structural Preferences and Vibrational Analysis of 2-Hydroxy-2-methyl-1-phenylpropan-1-one: A Computational and Infrared Spectroscopic Research
}

\author{
O. Belaidi, ${ }^{1}$ T. Bouchaour, ${ }^{1}$ and U. Maschke ${ }^{2}$ \\ ${ }^{1}$ Laboratoire de Recherche sur les Macromolécules, Faculté des Sciences, Université Abou Bekr Belkaid, BP119, 13000 Tlemcen, Algeria \\ ${ }^{2}$ Unité Matériaux et Transformations (UMET), UMR CNRS N8207, Université Lille 1-Sciences et Technologies, \\ 59655 Villeneuve d'Ascq Cedex, France
}

Correspondence should be addressed to O. Belaidi; othmanblaidi@gmail.com

Received 13 February 2013; Revised 6 May 2013; Accepted 11 May 2013

Academic Editor: Elsa Caetano

Copyright ( $\odot 2013$ O. Belaidi et al. This is an open access article distributed under the Creative Commons Attribution License, which permits unrestricted use, distribution, and reproduction in any medium, provided the original work is properly cited.

\begin{abstract}
The Fourier transform infrared (FTIR) spectrum of 2-hydroxy-2-methyl-1-phenylpropan-1-one has been measured in the region $4000-700 \mathrm{~cm}^{-1}$. The most stable conformation of title molecule was found after a careful potential energy surfaces study. The molecular geometry, vibrational frequencies, and infrared intensities have been calculated by using ab initio HF and density functional theory calculation B3LYP with $6-311+G^{* * *}$ basis set. Scaled frequencies and potential energy distribution were calculated for band assignment. We found an excellent agreement between the experimental and the simulated spectra. Energy gap between HOMO and LUMO explains the eventual charge transfer interactions taking place within the molecule.
\end{abstract}

\section{Introduction}

The radiation curing industry is one of the most rapidly developing fields in the entire coating industry. The low toxicity, cheapness, speed, control, and ease of formulation and operation are some of the main advantages of this growing technology. UV and/or visible light radiation is used to induce photochemical polymerization or cross-linking of a monomer, oligomer, or prepolymer formulation containing a certain type of unsaturation, such as an acrylic group, and an appropriate initiator $[1,2]$. Among the large group of known photoinitiator structures, hydroxyalkylphenones, such as 2-hydroxy-2-methyl-1-phenylpropan-1-one (Figure 1), have gained much interest due to their high reactivity and universal applicability $[3,4]$.

Despite the high practical value of title molecule, its physicochemical properties have been relatively little looked into [8-12].

To our best knowledge no structural data and detailed interpretation of the vibrational spectra of the title molecule are presented in the literature. This prompted us to look into the vibrational spectroscopy of the title molecule more carefully.

\section{Experimental}

2-Hydroxy-2-methyl-1-phenylpropan-1-one (purity 97\%) was purchased from Sigma-Aldrich and used as such without further purification. The infrared spectra of liquid films placed between $\mathrm{KBr}$ pellets were recorded within 4000$700 \mathrm{~cm}^{-1}$ range with a Perkin Elmer FTIR System-2000 model.

\section{Computational}

Potential energy curves were obtained by calculating the variation of total energy of title molecule with dihedral angles. For this purpose the calculations were performed with B3LYP functional and 6-311+G** basis set. The dihedral angles were varied from $0^{\circ}$ to $360^{\circ}$ with an interval of $10^{\circ}$. 
b3lyp 6-311+Gdp D1M7M12M5.out

Created by gauss view 3.09

12/05/2013 09:53:55

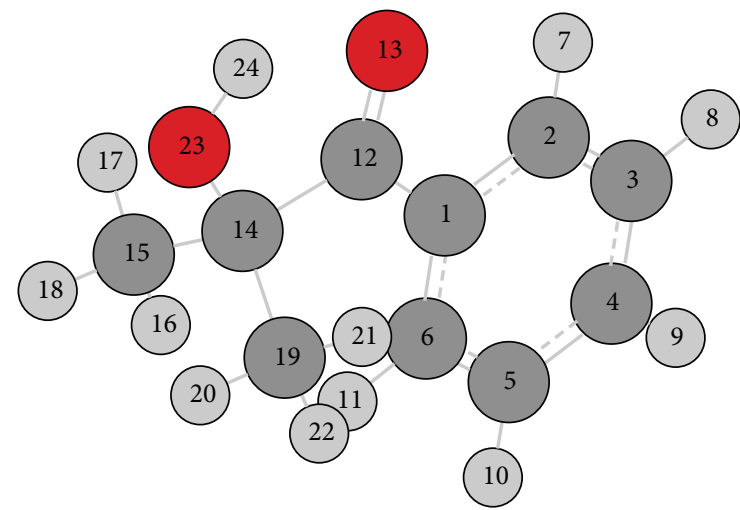

FIGURE 1: The optimized geometrical structure with atoms numbering of 2-hydroxy-2-methyl-1-phenylpropan-1-one.

All DFT calculations were performed using Becke's 3 parameter (local, nonlocal, Hartree-Fock) hybrid exchange functionals with Lee-Yang-parr correlational functionals (B3LYP) $[13,14]$.

The harmonic vibrational frequencies of the stable conformation were calculated at the same level of theories used for the calculated optimized geometry. Two levels of theory were used for vibrational analysis B3LYP/6-311 $+\mathrm{G}^{* *}$ and $\mathrm{HF} / 6-311+\mathrm{G}^{* *}$; the calculated frequencies were scaled down by the wavenumber linear scaling (WLS) procedure of Yoshida et al. $[15,16]$ using the following equation, for DFT:

$$
\frac{v_{\text {obs }}}{v_{\text {calc }}}=1.0087-0.0000163 v_{\text {calc }}
$$

and the scaling factor proposed by Baker et al. [17] for HF. The scaling procedures are used due to the fact that the anharmonic effects are neglected when calculating wavenumbers at HF and DFT theories, which make the calculated value higher than the experimental one. All the calculations were carried out with the Gaussian 03 program [18]. The vibrational assignments of normal modes were provided on the basis of PED calculation using the program GAR2PED [19].

\section{Conformational Analysis}

Due to the flexibility of 2-hydroxy-2-methyl-1-phenylpropan1-one and possible rotational isomerism, we examined the variation of energy with respect to $\mathrm{C}_{6} \mathrm{C}_{1} \mathrm{C}_{12} \mathrm{C}_{14}$, $\mathrm{C}_{1} \mathrm{C}_{12} \mathrm{C}_{14} \mathrm{C}_{23}$, and $\mathrm{C}_{12} \mathrm{C}_{14} \mathrm{C}_{23} \mathrm{O}_{24}$ dihedral angles. From Figure 2 we see that the energy is increasing when increasing dihedral angle to a maximum in energy of $-3.2510^{5} \mathrm{kcal} / \mathrm{mol}$ at $180^{\circ}$. There is only one minimum at $0^{\circ}$ with an energy of $-3.3810^{5} \mathrm{kcal} / \mathrm{mol}$.

It is well seen from Figures 3 and 4 that the molecule takes two minima at $0^{\circ}$ and $180^{\circ}$. In Figure 3 the difference in energy between the two minima is $419.7 \mathrm{kcal} / \mathrm{mol}$, and it is $8.785 \mathrm{kcal} / \mathrm{mol}$ in Figure 4.

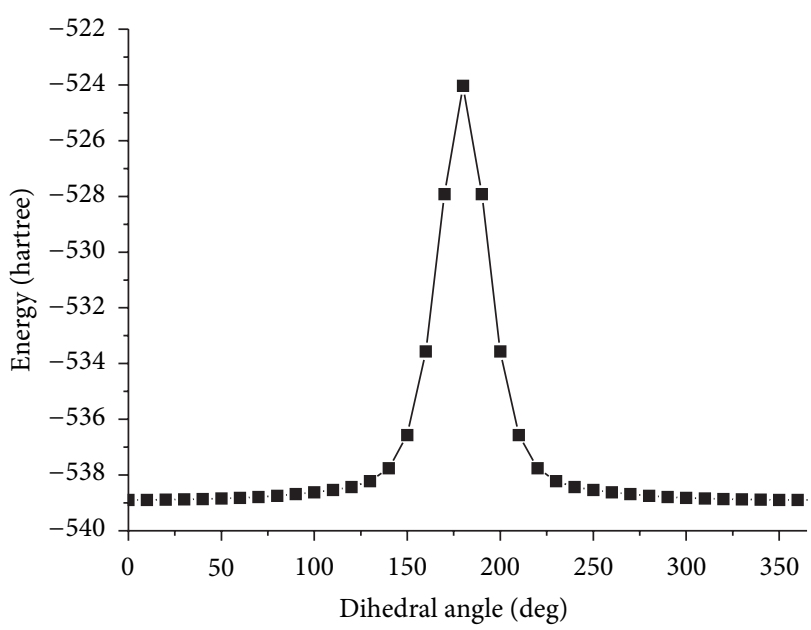

Figure 2: Potential energy curves of 2-hydroxy-2-methyl-1-

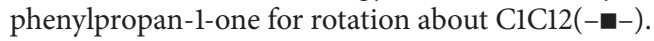

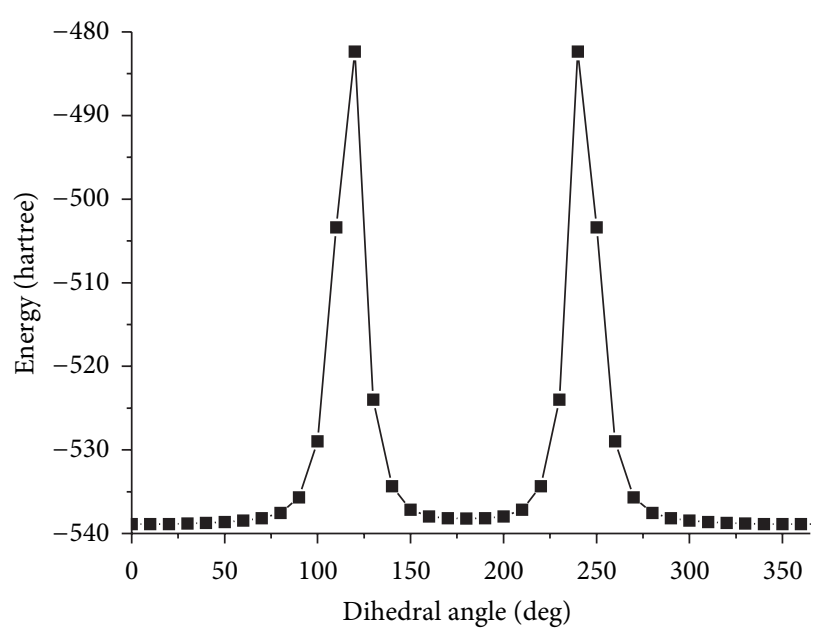

FIGURE 3: Potential energy curves of 2-hydroxy-2-methyl-1phenylpropan-1-one for rotation about $\mathrm{C} 12 \mathrm{C} 14(-\mathbf{-}-)$.

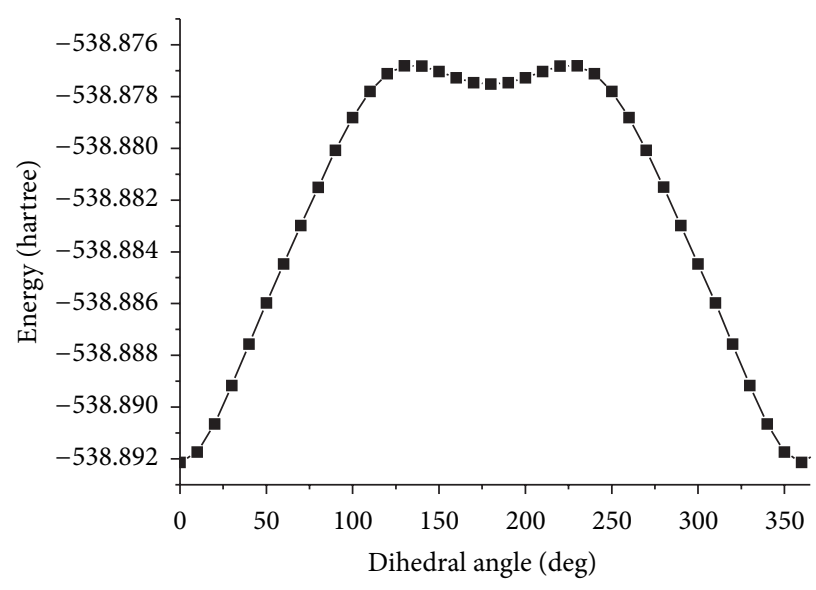

FIGURE 4: Potential energy curves of 2-hydroxy-2-methyl-1phenylpropan-1-one for rotation about C14O23(-匹-). 
The potential energy surfaces revealed the existence of tree conformers. The energy of the most stable conformation is $-3.3810^{5} \mathrm{kcal} / \mathrm{mol}$.

\section{Geometry Optimizations}

The most stable structure is recognized as true minima due to the lack of imaginary harmonic frequencies. The geometry parameters are represented in Table 1 . To the best of our knowledge, exact experimental data of the geometrical parameters of the 2-hydroxy-2-methyl-1-phenylpropan-1-one are not available in the literature. Therefore our optimized structural parameters are compared with the XRD data of closely related molecules benzoic acid and butyric acid $[5,6]$. The theoretical results are almost comparable with the experimental XRD values. The carbon-oxygen $\mathrm{C}_{14} \mathrm{O}_{23}$ $=1.421$ and carbon-carbon $\mathrm{C}_{12} \mathrm{C}_{14}=1.554, \mathrm{C}_{14} \mathrm{C}_{15}=1.540$, and $\mathrm{C} 14 \mathrm{C} 19=1.540$ bond lengths are in agreement with the XRD values [7] 1.38, 1.55, 1.54, and 1.54, respectively. In the phenyl ring, the bond lengths and the bond angles are in good agreement with the experimental one [7]. Thus the ring is slightly distorted and smaller than $120^{\circ}$ at the point of substitution.

In Table 1, a small difference between the two levels of theory HF and DFT should be noticed for the calculated coordinates. The bond lengths are less estimated by $\mathrm{HF}$ with a maximum difference of $0.01 \mathrm{~A}^{\circ}$. However, this difference is about $0.02 \mathrm{~A}^{\circ}$ for $\mathrm{C} 14 \mathrm{O} 23$ bond. The B3LYP calculations lead to a planar structure of the phenyl ring, ketone and hydroxyl groups. Otherwise, these groups lie in a near planar form according to the $\mathrm{HF}$ optimization. This could be seen directly from dihedral angles $\left(\mathrm{C}_{6} \mathrm{C}_{1} \mathrm{C}_{12} \mathrm{O}_{13}\right.$, $\mathrm{C}_{6} \mathrm{C}_{1} \mathrm{C}_{12} \mathrm{C}_{14}, \mathrm{C}_{1} \mathrm{C}_{12} \mathrm{C}_{14} \mathrm{C}_{15}, \mathrm{C}_{1} \mathrm{C}_{12} \mathrm{C}_{14} \mathrm{C}_{19}, \mathrm{C}_{1} \mathrm{C}_{12} \mathrm{C}_{14} \mathrm{O}_{23}$, and $\mathrm{C}_{12} \mathrm{C}_{14} \mathrm{O}_{23} \mathrm{H}_{24}$ ) deviating about $10^{\circ}$ from those obtained by B3LYP level of theory.

The zero point corrected energy for the most stable structure of the title molecule is $-337.91110^{3} \mathrm{kcal} / \mathrm{mol}$ at B3LYP/6$311+\mathrm{G}^{* *}$ and $-335.78610^{3} \mathrm{kcal} / \mathrm{mol}$ at $\mathrm{HF} / 6-311+\mathrm{G}^{* *}$. The energy calculated by DFT is lower showing more stability than that calculated at HF method.

\section{Vibrational Analysis}

The title compound contains 24 atoms leading to 66 normal vibrational modes. We compared the experimental IR spectrum with the computed IR spectrum, and we checked whether we can recognize all the different bands. Table 2 represents the calculated and scaled fundamental wavenumbers, intensities of vibrational peaks, and PED calculations along the internal coordinates obtained by B3LYP/6-311+G ${ }^{* *}$ level of theory. The corresponding experimental wavenumbers together with assignments and potential energy distribution are also reported in this table. Assignments have been made on the basis of PED, and they are given as per the internal coordinate system recommended by Pulay et al. using DFT [20]. The experimental and theoretical spectra are shown in Figure 5.
TABLE 1: Optimized geometry of 2-hydroxy-2-methyl-1-phenylpropan-1-one calculated at $\mathrm{HF}$ and B3LYP using $6-311+\mathrm{G}^{* *}$ basis set.

\begin{tabular}{|c|c|c|c|}
\hline \multirow{2}{*}{$\begin{array}{l}\text { Internal } \\
\text { coordinates }\end{array}$} & \multicolumn{3}{|c|}{ Level of theory } \\
\hline & $\mathrm{RHF} / 6-311+\mathrm{G}^{* *}$ & $\mathrm{DFT} / 6-311+\mathrm{G}^{* *}$ & $\mathrm{X}$-ray ${ }^{\mathrm{a}}$ \\
\hline \multicolumn{4}{|c|}{ Bond lengths $(\AA)$} \\
\hline $\mathrm{C}_{1} \mathrm{C}_{2}$ & 1.395 & 1.405 & 1.39 \\
\hline $\mathrm{C}_{2} \mathrm{C}_{3}$ & 1.381 & 1.388 & 1.41 \\
\hline $\mathrm{C}_{3} \mathrm{C}_{4}$ & 1.386 & 1.395 & 1.37 \\
\hline $\mathrm{C}_{4} \mathrm{C}_{5}$ & 1.383 & 1.392 & 1.36 \\
\hline $\mathrm{C}_{5} \mathrm{C}_{6}$ & 1.385 & 1.392 & 1.42 \\
\hline $\mathrm{C}_{6} \mathrm{C}_{1}$ & 1.390 & 1.402 & 1.39 \\
\hline $\mathrm{CH}_{\text {(ring) average }}$ & 1.073 & 1.082 & \\
\hline $\mathrm{C}_{1} \mathrm{C}_{12}$ & 1.506 & 1.499 & 1.48 \\
\hline $\mathrm{C}_{12} \mathrm{O}_{13}$ & 1.194 & 1.221 & 1.24 \\
\hline $\mathrm{C}_{12} \mathrm{C}_{14}$ & 1.547 & 1.554 & $1.55^{\mathrm{b}}$ \\
\hline $\mathrm{C}_{14} \mathrm{C}_{15}$ & 1.535 & 1.540 & $1.54^{\mathrm{b}}$ \\
\hline $\mathrm{C}_{15} \mathrm{H}_{16}$ & 1.082 & 1.090 & \\
\hline $\mathrm{C}_{15} \mathrm{H}_{17}$ & 1.085 & 1.092 & \\
\hline $\mathrm{C}_{15} \mathrm{H}_{18}$ & 1.084 & 1.092 & \\
\hline $\mathrm{C}_{14} \mathrm{C}_{19}$ & 1.531 & 1.540 & $1.54^{\mathrm{b}}$ \\
\hline $\mathrm{C}_{19} \mathrm{H}_{20}$ & 1.084 & 1.092 & \\
\hline $\mathrm{C}_{19} \mathrm{H}_{21}$ & 1.085 & 1.092 & \\
\hline $\mathrm{C}_{19} \mathrm{H}_{22}$ & 1.081 & 1.090 & \\
\hline $\mathrm{C}_{14} \mathrm{O}_{23}$ & 1.399 & 1.421 & $1.38^{\mathrm{b}}$ \\
\hline $\mathrm{O}_{23} \mathrm{H}_{24}$ & 0.944 & 0.971 & 0.95 \\
\hline \multicolumn{4}{|c|}{ Bond angles $\left({ }^{\circ}\right)$} \\
\hline $\mathrm{C}_{1} \mathrm{C}_{2} \mathrm{C}_{3}$ & 120.8 & 120.9 & 120 \\
\hline $\mathrm{C}_{2} \mathrm{C}_{3} \mathrm{C}_{4}$ & 119.9 & 120.0 & 122 \\
\hline $\mathrm{C}_{3} \mathrm{C}_{4} \mathrm{C}_{5}$ & 119.7 & 119.7 & 118 \\
\hline $\mathrm{C}_{4} \mathrm{C}_{5} \mathrm{C}_{6}$ & 120.1 & 120.2 & 123 \\
\hline $\mathrm{C}_{5} \mathrm{C}_{6} \mathrm{C}_{1}$ & 120.6 & 120.6 & 118 \\
\hline $\mathrm{C}_{6} \mathrm{C}_{1} \mathrm{C}_{2}$ & 118.5 & 118.4 & 119 \\
\hline $\mathrm{CCH}_{\text {ring average }}$ & 119.4 & 119.3 & \\
\hline $\mathrm{C}_{6} \mathrm{C}_{1} \mathrm{C}_{12}$ & 125.1 & 124.8 & \\
\hline $\mathrm{C}_{1} \mathrm{C}_{12} \mathrm{O}_{13}$ & 118.7 & 119.2 & 118 \\
\hline $\mathrm{C}_{1} \mathrm{C}_{12} \mathrm{C}_{14}$ & 124.0 & 123.9 & \\
\hline $\mathrm{C}_{12} \mathrm{C}_{14} \mathrm{C}_{15}$ & 110.5 & 111.3 & \\
\hline $\mathrm{C}_{14} \mathrm{C}_{15} \mathrm{H}_{16}$ & 113.6 & 113.4 & \\
\hline $\mathrm{C}_{14} \mathrm{C}_{15} \mathrm{H}_{17}$ & 110.0 & 110.1 & \\
\hline $\mathrm{C}_{14} \mathrm{C}_{15} \mathrm{H}_{18}$ & 108.0 & 107.9 & \\
\hline $\mathrm{C}_{12} \mathrm{C}_{14} \mathrm{C}_{19}$ & 112.3 & 111.3 & \\
\hline $\mathrm{C}_{14} \mathrm{C}_{19} \mathrm{H}_{20}$ & 107.9 & 107.9 & \\
\hline $\mathrm{C}_{14} \mathrm{C}_{19} \mathrm{H}_{21}$ & 110.2 & 110.1 & \\
\hline $\mathrm{C}_{14} \mathrm{C}_{19} \mathrm{H}_{22}$ & 113.4 & 113.4 & \\
\hline $\mathrm{C}_{12} \mathrm{C}_{14} \mathrm{O}_{23}$ & 107.4 & 107.2 & \\
\hline $\mathrm{C}_{14} \mathrm{O}_{23} \mathrm{H}_{24}$ & 109.0 & 106.2 & 111 \\
\hline \multicolumn{4}{|c|}{ Dihedral angles $\left({ }^{\circ}\right)$} \\
\hline $\mathrm{C}_{1} \mathrm{C}_{2} \mathrm{C}_{3} \mathrm{C}_{4}$ & 0.4 & 0.0 & \\
\hline $\mathrm{C}_{2} \mathrm{C}_{3} \mathrm{C}_{4} \mathrm{C}_{5}$ & -0.1 & 0.0 & \\
\hline $\mathrm{C}_{3} \mathrm{C}_{4} \mathrm{C}_{5} \mathrm{C}_{6}$ & -0.2 & 0.0 & \\
\hline
\end{tabular}


TABLE 1: Continued.

\begin{tabular}{|c|c|c|c|}
\hline \multirow{2}{*}{$\begin{array}{l}\text { Internal } \\
\text { coordinates }\end{array}$} & \multicolumn{3}{|c|}{ Level of theory } \\
\hline & $\mathrm{RHF} / 6-311+\mathrm{G}^{* *}$ & DFT/6-311+G & $\mathrm{X}-$ ray $^{\mathrm{a}}$ \\
\hline $\mathrm{C}_{6} \mathrm{C}_{4} \mathrm{C}_{2} \mathrm{H}_{7}$ & 179.7 & 180.0 & \\
\hline $\mathrm{C}_{1} \mathrm{C}_{2} \mathrm{C}_{3} \mathrm{H}_{8}$ & -179.6 & 180.0 & \\
\hline $\mathrm{C}_{2} \mathrm{C}_{3} \mathrm{C}_{4} \mathrm{H}_{9}$ & 179.8 & 180.0 & \\
\hline $\mathrm{C}_{3} \mathrm{C}_{4} \mathrm{C}_{5} \mathrm{H}_{10}$ & 179.7 & 180.0 & \\
\hline $\mathrm{C}_{4} \mathrm{C}_{5} \mathrm{C}_{6} \mathrm{H}_{11}$ & 179.9 & 180.0 & \\
\hline $\mathrm{C}_{5} \mathrm{C}_{6} \mathrm{C}_{1} \mathrm{C}_{12}$ & 179.2 & -179.9 & \\
\hline $\mathrm{C}_{6} \mathrm{C}_{1} \mathrm{C}_{12} \mathrm{O}_{13}$ & -169.4 & 179.9 & \\
\hline $\mathrm{C}_{6} \mathrm{C}_{1} \mathrm{C}_{12} \mathrm{C}_{14}$ & 10.7 & 0.0 & \\
\hline $\mathrm{C}_{1} \mathrm{C}_{12} \mathrm{C}_{14} \mathrm{C}_{15}$ & -71.3 & -63.2 & \\
\hline $\mathrm{C}_{12} \mathrm{C}_{14} \mathrm{C}_{15} \mathrm{H}_{16}$ & 67.1 & 68.2 & \\
\hline $\mathrm{C}_{12} \mathrm{C}_{14} \mathrm{C}_{15} \mathrm{H}_{17}$ & -54.6 & -53.7 & \\
\hline $\mathrm{C}_{12} \mathrm{C}_{14} \mathrm{C}_{15} \mathrm{H}_{18}$ & -172.2 & -171.3 & \\
\hline $\mathrm{C}_{1} \mathrm{C}_{12} \mathrm{C}_{14} \mathrm{C}_{19}$ & 55.4 & 63.2 & \\
\hline $\mathrm{C}_{12} \mathrm{C}_{14} \mathrm{C}_{19} \mathrm{H}_{20}$ & 169.6 & 171.3 & \\
\hline $\mathrm{C}_{12} \mathrm{C}_{14} \mathrm{C}_{19} \mathrm{H}_{21}$ & 52.1 & 53.7 & \\
\hline $\mathrm{C}_{12} \mathrm{C}_{14} \mathrm{C}_{19} \mathrm{H}_{22}$ & -69.9 & -68.2 & \\
\hline $\mathrm{C}_{1} \mathrm{C}_{12} \mathrm{C}_{14} \mathrm{O}_{23}$ & 171.8 & -179.9 & \\
\hline $\mathrm{C}_{12} \mathrm{C}_{14} \mathrm{O}_{23} \mathrm{H}_{24}$ & 11.2 & 0.0 & \\
\hline
\end{tabular}

${ }^{\mathrm{a}} \mathrm{XRD}$ data of closely related molecules benzoic acid and butyric acid $[5,6]$. ${ }^{\mathrm{b}}$ XRD data of 4-butyl-1-(4-hydroxyphenyl)-2-phenyl-3,5-pyrazolidinedione [7].

6.1. Phenyl Ring Vibrations. The substituted-like molecule gives rise to $\mathrm{CH}$ stretching, $\mathrm{CH}$ in-plane and out-of-plane bending vibrations. The heteroaromatic structure shows the presence of $\mathrm{CH}$ stretching vibration in the region 3100$3000 \mathrm{~cm}^{-1}$ which is the characteristic region for the ready identification of $\mathrm{CH}$ stretching vibration $[15,21]$. In this region the bands are not affected appreciably by the nature of the substituent. The aromatic $\mathrm{CH}$ stretching frequencies rise from the modes observed at 3062, 3047, 3060, and $3080 \mathrm{~cm}^{-1}$ of benzene and its derivatives [22].

In this work, the $\mathrm{CH}$ stretching vibrations are observed at $3025,3060,3070,3090$, and $3108 \mathrm{~cm}^{-1}$ in the FTIR. The calculated values of these modes for the title molecule have been found to be 3031, 3041,3051,3066, and $3085 \mathrm{~cm}^{-1}$ at the B3LYP/6-311+G** level of theory. The calculated PEDs for these normal modes are greater than $85 \%$ (see Table 2).

The $\mathrm{CH}$-in plane bending vibrations usually occur in the region $1430-990 \mathrm{~cm}^{-1}$ and are very useful for characterization purposes. The band due to $\mathrm{CH}$-in plane ring vibrations interacting somewhat with $\mathrm{CC}$ stretching vibration is observed as a number of medium-to-weak sharp bands in the region of $1300-1000 \mathrm{~cm}^{-1}[23,24]$. In this study, the FTIR peaks at $1077,1170,1192$, and $1315 \mathrm{~cm}^{-1}$ are assigned to $\mathrm{CH}$ in-plane bending vibrations as reported in Table 2 . The strong peak below $900 \mathrm{~cm}^{-1}$ indicates clearly the aromatic aspect of the molecule. Substitution patterns on the ring can be judged from the $\mathrm{CH}$ out-of-plane bending in the region $900-650 \mathrm{~cm}^{-1}$, and these bands are highly informative [25]. The $\mathrm{CH}$ out-of-plane bending vibrations are strongly coupled vibrations in the region $900-667 \mathrm{~cm}^{-1}$ [26]. In the present

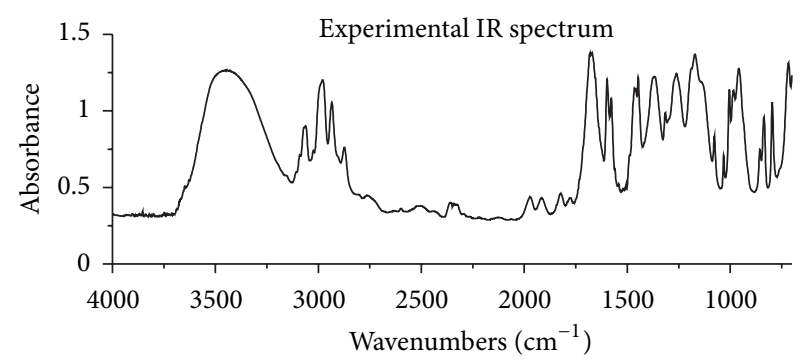

(a)

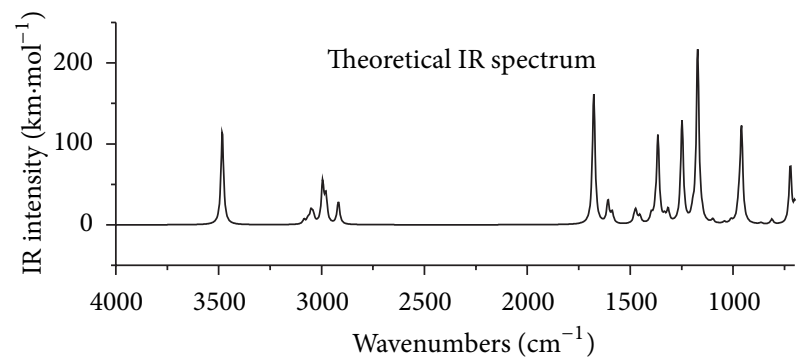

(b)

FIgURE 5: Experimental and theoretical infrared spectra of 2hydroxy-2-methyl-1-phenylpropan-1-one.

study, the calculated frequencies at 994, 950, 857, 721, and $697 \mathrm{~cm}^{-1}$ may be assigned to the experimental bands at 934, 836 , and $716 \mathrm{~cm}^{-1}$. The calculated PEDs show that these bands are not pure $\mathrm{CH}$ out-of-plane bending vibrations.

The ring carbon-carbon stretching vibration occurs in the region $1625-1430 \mathrm{~cm}^{-1}$. In general, the bands are of variable intensity and are observed at 1625-1590, 1590-1575, 1540$1470,1460-1430$, and $1380-1280 \mathrm{~cm}^{-1}$ from the frequency ranges given by Varsanyi [27] for the five bands in the region. In this work, the frequencies observed in the FTIR spectrum at $1598,1578,1489$, and $1447 \mathrm{~cm}^{-1}$ are well reproduced by the theoretical frequencies at 1607, 1587, 1497, and $1452 \mathrm{~cm}^{-1}$. The calculated PEDs show that these modes are not pure CC stretching vibrations. The normal mode no. 31 is attributed to the ring breathing vibration; the calculated PED for this mode is $61 \%$.

6.2. The CC Vibrations. The CC stretching vibrations of the title molecule occur in the region from 1000 to $1600 \mathrm{~cm}^{-1}$. The majority of these modes of vibration are combined with $\mathrm{CH}$ and $\mathrm{OH}$ bending vibrations. The calculated $\mathrm{PED}$ for $\mathrm{CC}$ stretching are of about $13 \%$ for normal modes \#37, \#38, and \#39. However the normal mode \#40 is attributed to the CC stretching vibrations with a potential of $37 \%$ and less than $10 \%$ for normal modes \#51 and \#53. The CCC in-plane bending frequencies are found at 372, 443, 529, 618, 701, 860, and $923 \mathrm{~cm}^{-1}$.

The CC torsions are likely to occur in the region below $500 \mathrm{~cm}^{-1}$. For the title molecule, we found five normal modes representing the torsion about the bond $\mathrm{C}_{12} \mathrm{C}_{14}$. The calculated wavenumbers are 22, 79, 163, 265, and $325 \mathrm{~cm}^{-1}$. The calculated PEDs are about $50 \%$. These modes are not pure 
TABLE 2: Frequencies and PEDs for 2-hydroxy-2-methyl-1-phenylpropan-1-one molecule.

\begin{tabular}{|c|c|c|c|c|c|c|}
\hline \multirow[b]{2}{*}{$\mathrm{b}$} & \multirow[b]{2}{*}{ Observed $\left(\mathrm{cm}^{-1}\right)$} & \multicolumn{3}{|c|}{$\mathrm{DFT} / 6-311+\mathrm{G}^{* *}$} & \multirow{2}{*}{$\begin{array}{l}\mathrm{HF} / 6-311+\mathrm{G}^{* *} \\
\text { Scaled }\left(\mathrm{cm}^{-1}\right)\end{array}$} & \multirow{2}{*}{ Assignment $(\% \mathrm{PED})^{\mathrm{a}}$} \\
\hline & & $\begin{array}{l}\text { Unscaled } \\
\left(\mathrm{cm}^{-1}\right)\end{array}$ & $\begin{array}{l}\text { Scaled } \\
\left(\mathrm{cm}^{-1}\right)\end{array}$ & $\begin{array}{l}\text { Intensity } \\
\left(\mathrm{km} \cdot \mathrm{mol}^{-1}\right)\end{array}$ & & \\
\hline 1 & - & 22.74 & 22.92 & 0.0053 & 17.23 & $\tau\left(\mathrm{C}_{12} \mathrm{C}_{14}\right)(68), \mathrm{o}\left(\mathrm{C}_{12} \mathrm{C}_{13}\right)(22)$ \\
\hline 2 & - & 78.66 & 79.24 & 1.2078 & 72.16 & $\tau\left(\mathrm{C}_{12} \mathrm{C}_{14}\right)(48), \mathrm{o}\left(\mathrm{C}_{14} \mathrm{O}_{23}\right)(26), \tau\left(\mathrm{C}_{14} \mathrm{C}_{23}\right)$ \\
\hline 3 & - & 137.69 & 174.71 & 0.1931 & 133.22 & $\tau\left(\mathrm{CH}_{3}\right)(63), \mathrm{o}\left(\mathrm{C}_{12} \mathrm{O}_{13}\right)(25)$ \\
\hline 4 & - & 162.19 & 163.17 & 0.1275 & 161.76 & $\tau\left(\mathrm{C}_{12} \mathrm{C}_{14}\right)(89)$ \\
\hline 5 & - & 184.98 & 186.03 & 0.0007 & 190.12 & $\tau\left(\mathrm{CH}_{3}\right)(76)$ \\
\hline 6 & - & 215.31 & 216.42 & 0.0829 & 218.35 & $\tau\left(\mathrm{CH}_{3}\right)(62), \tau\left(\mathrm{C}_{12} \mathrm{C}_{14}\right)(27)$ \\
\hline 7 & - & 257.12 & 258.28 & 0.2225 & 258.41 & $\tau\left(\mathrm{CH}_{3}\right)(63), \rho\left(\mathrm{C}_{14} \mathrm{O}_{23}\right)(14)$ \\
\hline 8 & - & 264.22 & 265.38 & 1.0193 & 261.87 & $\begin{array}{l}\tau\left(\mathrm{C}_{12} \mathrm{C}_{14}\right)(54), \delta\left(\mathrm{C}_{12} \mathrm{C}_{14} \mathrm{C}_{19}\right)(25), \delta\left(\mathrm{C}_{12} \mathrm{C}_{14} \mathrm{C}_{15}\right) \\
(14)\end{array}$ \\
\hline 9 & - & 324.53 & 325.64 & 2.6572 & 318.84 & $\tau\left(\mathrm{C}_{12} \mathrm{C}_{14}\right)(54), \delta\left(\mathrm{C}_{12} \mathrm{C}_{14} \mathrm{C}_{19}\right)$ \\
\hline 10 & - & 371.29 & 372.27 & 4.4029 & 363.0228 & $\begin{array}{l}\delta\left(\mathrm{C}_{12} \mathrm{C}_{14} \mathrm{C}_{15}\right)(53), \delta\left(\mathrm{C}_{12} \mathrm{C}_{14} \mathrm{C}_{19}\right)(26), \delta\left(\mathrm{C}_{1} \mathrm{C}_{12} \mathrm{C}_{14}\right) \\
(14)\end{array}$ \\
\hline 11 & - & 415.70 & 416.5 & 12.5757 & 368.45 & $\mathrm{o}\left(\mathrm{O}_{23} \mathrm{H}\right)(51), \tau\left(\mathrm{C}_{14} \mathrm{O}_{23}\right)$ \\
\hline 12 & - & 417.85 & 418.64 & 13.1148 & 418.74 & $\mathrm{o}\left(\mathrm{O}_{23} \mathrm{H}\right)(37), \tau\left(\mathrm{C}_{14} \mathrm{O}_{23}\right)(34), \mathrm{o}\left(\mathrm{O}_{13} \mathrm{C}_{12}\right)(16)$ \\
\hline 13 & - & 442.58 & 443.24 & 23.2077 & 425.91 & $\delta\left(\mathrm{C}_{13} \mathrm{C}_{12} \mathrm{C}_{14}\right)(79), \delta\left(\mathrm{C}_{12} \mathrm{C}_{14} \mathrm{C}_{15}\right)$ \\
\hline 14 & - & 444.60 & 445.25 & 39.0992 & 436.6872 & $\mathrm{o}\left(\mathrm{O}_{23} \mathrm{H}\right)(37), \mathrm{o}\left(\mathrm{C}_{1} \mathrm{C}_{12} \mathrm{O}_{13}\right)(28), \rho\left(\mathrm{C}_{12} \mathrm{C}_{14} \mathrm{O}_{23}\right)(23)$ \\
\hline 15 & - & 506.39 & 506.62 & 13.7159 & 477.5168 & $\mathrm{o}\left(\mathrm{O}_{23} \mathrm{H}\right)(69), \rho\left(\mathrm{C}_{12} \mathrm{C}_{14} \mathrm{O}_{23}\right)(20)$ \\
\hline 16 & - & 529.30 & 529.34 & 0.8797 & 524.676 & $\delta\left(\mathrm{O}_{13} \mathrm{C}_{12} \mathrm{C}_{14}\right)(48), \delta\left(\mathrm{C}_{1} \mathrm{C}_{12} \mathrm{C}_{14}\right)(45)$ \\
\hline 17 & - & 619.59 & 618.72 & 2.1280 & 611.3124 & $\begin{array}{l}\delta\left(\mathrm{C}_{12} \mathrm{C}_{14} \mathrm{C}_{19}\right)(44), \delta\left(\mathrm{C}_{12} \mathrm{C}_{14} \mathrm{C}_{15}\right)(24) \\
\delta\left(\mathrm{O}_{12} \mathrm{C}_{13} \mathrm{C}_{14}\right)(15)\end{array}$ \\
\hline 18 & - & 635.52 & 634.45 & 1.5297 & 623.346 & Ring deformation (79), $\delta\left(\mathrm{C}_{1} \mathrm{C}_{12} \mathrm{C}_{13}\right)(17)$ \\
\hline 19 & - & 699.33 & 697.44 & 13.7753 & 689.55 & $\mathrm{o}(\mathrm{CH})_{\text {ring }}(62), \delta\left(\mathrm{C}_{12} \mathrm{C}_{14} \mathrm{O}_{23}\right)(21)$ \\
\hline 20 & - & 703.85 & 701.89 & 11.8256 & 693.76 & $\delta\left(\mathrm{C}_{1} \mathrm{C}_{12} \mathrm{C}_{14}\right)(68), \delta\left(\mathrm{C}_{12} \mathrm{C}_{14} \mathrm{C}_{15}\right)$ \\
\hline 21 & 716 & 723.68 & 721.43 & 72.3248 & 730.69 & $\mathrm{o}\left(\mathrm{C}_{19} \mathrm{H}_{3}\right)(53), \mathrm{o}(\mathrm{CH})_{\text {ring }}(23) \tau\left(\mathrm{C}_{12} \mathrm{C}_{14}\right)(17)$ \\
\hline 22 & 796 & 811.44 & 807.76 & 6.0346 & 815.01 & $\begin{array}{l}\mathrm{o}\left(\mathrm{C}_{15} \mathrm{H}_{3}\right)(44), \delta\left(\mathrm{O}_{13} \mathrm{C}_{12} \mathrm{C}_{14}\right)(24), \delta\left(\mathrm{C}_{6} \mathrm{C}_{1} \mathrm{C}_{12}\right)(15), \\
\mathrm{o}\left(\mathrm{O}_{13} \mathrm{C}_{12}\right)(12)\end{array}$ \\
\hline 23 & 836 & 862.56 & 857.93 & 0.2930 & 863.31 & $\mathrm{o}(\mathrm{CH})_{\text {ring }}(98)$ \\
\hline 24 & 856 & 865.50 & 860.82 & 1.4073 & 877.23 & $\delta\left(\mathrm{C}_{12} \mathrm{C}_{14} \mathrm{C}_{19}\right)(67), \delta\left(\mathrm{O}_{13} \mathrm{C}_{12} \mathrm{C}_{14}\right)$ \\
\hline 25 & - & 929.00 & 923.01 & 0.0318 & 928.86 & $\begin{array}{l}\delta\left(\mathrm{C}_{12} \mathrm{C}_{14} \mathrm{O}_{23}\right)(41), \delta\left(\mathrm{C}_{12} \mathrm{C}_{14} \mathrm{C}_{15}\right)(29), \delta\left(\mathrm{C}_{12} \mathrm{C}_{14} \mathrm{C}_{19}\right) \\
(13)\end{array}$ \\
\hline 26 & 934 & 957.15 & 950.54 & 1.1541 & 958.57 & $\begin{array}{l}\delta\left(\mathrm{C}_{12} \mathrm{C}_{14} \mathrm{C}_{15}\right)(22), \rho\left(\mathrm{C}_{15} \mathrm{H}_{3}\right)(19), \rho\left(\mathrm{C}_{15} \mathrm{H}_{3}\right)(17), \mathrm{o} \\
(\mathrm{CH})_{\text {ring }}(14)\end{array}$ \\
\hline 27 & 959 & 966.31 & 959.49 & 119.3931 & 978.08 & $v\left(\mathrm{C}_{14} \mathrm{O}_{23}\right)(83)$ \\
\hline 28 & 984 & 980.27 & 973.13 & 19.8320 & 997.34 & $\begin{array}{l}\rho\left(\mathrm{C}_{19} \mathrm{H}_{3}\right)(24), \delta\left(\mathrm{C}_{1} \mathrm{C}_{12} \mathrm{C}_{14}\right)(24), v\left(\mathrm{C}_{14} \mathrm{O}_{23}\right)(22) \\
\rho\left(\mathrm{C}_{14} \mathrm{O}_{23} \mathrm{H}\right)(12)\end{array}$ \\
\hline 29 & - & 1002.21 & 994.55 & 0.2486 & 1000.37 & $\mathrm{o}(\mathrm{CH})_{\text {ring }}(53), \rho\left(\mathrm{C}_{15} \mathrm{H}_{3}\right)(23), \rho\left(\mathrm{C}_{19} \mathrm{H}_{3}\right)(21)$ \\
\hline 30 & 984 & 1016.36 & 1008.36 & 0.1061 & 1020.30 & $\rho\left(\mathrm{C}_{19} \mathrm{H}_{3}\right)(68), \rho\left(\mathrm{C}_{15} \mathrm{H}_{3}\right)(16)$ \\
\hline 31 & - & 1017.60 & 1009.57 & 3.5213 & 1026.49 & Ring breathing $(61), \delta\left(\mathrm{C}_{15} \mathrm{H}_{3}\right)(21)$ \\
\hline 32 & 1004 & 1022.17 & 1014.03 & 0.4101 & 1031.95 & $\mathrm{o}\left(\mathrm{C}_{19} \mathrm{H}_{3}\right)(59), \delta\left(\mathrm{C}_{15} \mathrm{H}_{3}\right)(28)$ \\
\hline 33 & 1034 & 1051.38 & 1042.50 & 2.1205 & 1034.20 & $\rho\left(\mathrm{C}_{15} \mathrm{H}_{3}\right)(61), \rho\left(19 \mathrm{H}_{3}\right)(18), \beta(\mathrm{CH})_{\text {ring }}(15)$ \\
\hline 34 & 1077 & 1108.61 & 1098.22 & 4.6450 & 1082.28 & $\beta(\mathrm{CH})_{\text {ring }}(72), \beta\left(\mathrm{C}_{15} \mathrm{H}_{3}\right)(12)$ \\
\hline 35 & 1134 & 1163.52 & 1151.57 & 4.8064 & 1116.30 & $\beta\left(\mathrm{C}_{14} \mathrm{O}_{23} \mathrm{H}\right)(64), \beta\left(\mathrm{C}_{19} \mathrm{H}_{3}\right)(26)$ \\
\hline 36 & - & 1182.42 & 1169.91 & 11.4215 & 1157.55 & $\mathrm{o}\left(\mathrm{C}_{12} \mathrm{C}_{14} \mathrm{O}_{23}\right)(48), \rho\left(\mathrm{C}_{15} \mathrm{H}_{3}\right)(36), \rho\left(\mathrm{C}_{19} \mathrm{H}_{3}\right)(14)$ \\
\hline 37 & 1170 & 1184.74 & 1172.16 & 164.1487 & 1186.76 & $\beta(\mathrm{CH})_{\text {ring }}(40), \delta\left(\mathrm{O}_{23} \mathrm{H}\right)(28), \nu\left(\mathrm{C}_{1} \mathrm{C}_{12}\right)$ \\
\hline 38 & - & 1185.47 & 1172.87 & 43.3932 & 1193.31 & $\rho\left(\mathrm{C}_{15} \mathrm{H}_{3}\right)(40), \delta\left(\mathrm{O}_{23} \mathrm{H}\right)(21), v\left(\mathrm{C}_{1} \mathrm{C}_{12}\right)$ \\
\hline 39 & 1192 & 1210.11 & 1196.76 & 10.7158 & 1210.98 & $\beta\left(\mathrm{C}_{15} \mathrm{H}_{3}\right)(31), \beta(\mathrm{CH})_{\text {ring }}(15), \nu\left(\mathrm{C}_{14} \mathrm{C}_{19}\right)(13)$ \\
\hline 40 & 1260 & 1263.08 & 1248.06 & 126.7838 & 1226.8 & $\nu\left(\mathrm{C}_{1} \mathrm{C}_{12}\right)(37), \beta\left(\mathrm{C}_{14} \mathrm{O}_{23} \mathrm{H}\right)(25), \nu\left(\mathrm{C}_{12} \mathrm{C}_{14}\right)(14)$ \\
\hline
\end{tabular}


TABLE 2: Continued.

\begin{tabular}{|c|c|c|c|c|c|c|}
\hline \multirow{2}{*}{$\mathrm{b}$} & \multirow[b]{2}{*}{ Observed $\left(\mathrm{cm}^{-1}\right)$} & \multicolumn{3}{|c|}{$\mathrm{DFT} / 6-311+\mathrm{G}^{* *}$} & \multirow{2}{*}{$\begin{array}{l}\mathrm{HF} / 6-311+\mathrm{G}^{* *} \\
\text { Scaled }\left(\mathrm{cm}^{-1}\right)\end{array}$} & \multirow{2}{*}{ Assignment $(\% \mathrm{PED})^{\mathrm{a}}$} \\
\hline & & $\begin{array}{c}\text { Unscaled } \\
\left(\mathrm{cm}^{-1}\right)\end{array}$ & $\begin{array}{l}\text { Scaled } \\
\left(\mathrm{cm}^{-1}\right)\end{array}$ & $\begin{array}{c}\text { Intensity } \\
\left(\mathrm{km} \cdot \mathrm{mol}^{-1}\right)\end{array}$ & & \\
\hline 41 & $1280 \mathrm{sh}$ & 1332.76 & 1315.40 & 15.7006 & 1273.70 & $\rho\left(\mathrm{C}_{19} \mathrm{H}_{3}\right)(49), \rho\left(\mathrm{C}_{15} \mathrm{H}_{3}\right)(27)$ \\
\hline 42 & 1315 & 1350.99 & 1332.99 & 6.7816 & 1339.85 & $\beta(\mathrm{CH})_{\text {ring }}(52), \delta\left(\mathrm{C}_{14} \mathrm{O}_{23} \mathrm{H}\right)(32)$, \\
\hline 43 & 1363 & 1385.14 & 1365.91 & 106.1420 & 1376.17 & $\beta\left(\mathrm{C}_{14} \mathrm{O}_{23} \mathrm{H}\right)(83)$ \\
\hline 44 & 1374 & 1397.17 & 1377.50 & 10.1008 & 1407.32 & $\delta_{\text {sym }}\left(\mathrm{C}_{19} \mathrm{H}_{3}\right)(52), \delta_{\text {sym }}\left(\mathrm{C}_{15} \mathrm{H}_{3}\right)$ \\
\hline 45 & 1387 & 1418.22 & 1397.77 & 9.5326 & 1427.11 & $\delta_{\text {sym }}\left(\mathrm{C}_{19} \mathrm{H}_{3}\right)(53), \delta_{\text {sym }}\left(\mathrm{C}_{15} \mathrm{H}_{3}\right)$ \\
\hline 46 & - & 1475.12 & 1452.48 & 0.6846 & 1463.36 & $\rho\left(\mathrm{C}_{19} \mathrm{H}_{3}\right)(88), \rho\left(\mathrm{C}_{15} \mathrm{H}_{3}\right)(8)$, \\
\hline 47 & 1447 & 1475.36 & 1452.71 & 7.9436 & 1466.61 & $\nu(\mathrm{CC})_{\text {ring }}(65), \delta\left(\mathrm{C}_{15} \mathrm{H}_{3}\right)(21)$ \\
\hline 48 & - & 1485.16 & 1462.12 & 1.4500 & 1477.58 & $\delta_{\text {asym }}\left(\mathrm{C}_{15} \mathrm{H}_{3}\right)(47), \delta_{\text {asym }}\left(\mathrm{C}_{19} \mathrm{H}_{3}\right)(41)$, \\
\hline 49 & 1457 & 1497.19 & 1473.67 & 14.4008 & 1487.97 & $\delta_{\text {asym }}\left(\mathrm{Cl}_{3} \mathrm{H}_{3}\right)(70)$ \\
\hline 50 & 1465 & 1504.27 & 1480.47 & 5.7803 & 1494.03 & $\delta_{\text {asym }}\left(\mathrm{C}_{19} \mathrm{H}_{3}\right)(75), \delta_{\text {asym }}\left(\mathrm{C}_{15} \mathrm{H}_{3}\right)(17)$ \\
\hline 51 & 1489 & 1521.61 & 1497.10 & 0.3870 & 1517.46 & $\nu(\mathrm{CC})_{\text {ring }}(51), \delta\left(\mathrm{C}_{15} \mathrm{H}_{3}\right)(21)$ \\
\hline 52 & 1578 & 1615.70 & 1587.20 & 13.0932 & 1619.02 & $\nu(\mathrm{CC})_{\text {ring }}(39), \delta\left(\mathrm{C}_{19} \mathrm{H}_{3}\right)(37), \delta\left(\mathrm{C}_{15} \mathrm{H}_{3}\right)(20)$ \\
\hline 53 & 1598 & 1636.91 & 1607.47 & 28.0759 & 1642.55 & $\nu(\mathrm{CC})_{\text {ring }}(65), \delta(\mathrm{CH})_{\text {ring }}(23)$ \\
\hline 54 & 1683 & 1710.11 & 1677.31 & 162.2382 & 1768.21 & $v\left(\mathrm{C}_{12} \mathrm{O}_{13}\right)(82)$ \\
\hline 55 & 2935 & 3042.17 & 2917.78 & 19.8324 & 2926.52 & $v_{\text {sym }} \mathrm{CH}$ of $\left(\mathrm{C}_{19} \mathrm{H}_{3}\right)(46), v_{\text {sym }} \mathrm{CH}$ of $\left(\mathrm{C}_{15} \mathrm{H}_{3}\right)$ (35) \\
\hline 56 & - & 3044.82 & 2920.19 & 8.9574 & 2931.07 & $v_{\text {sym }} \mathrm{CH}$ of $\left(\mathrm{C}_{19} \mathrm{H}_{3}\right)(50), v_{\text {sym }} \mathrm{CH}$ of $\left(\mathrm{C}_{15} \mathrm{H}_{3}\right)$ \\
\hline 57 & - & 3107.42 & 2977.06 & 8.1447 & 2985.04 & $v_{\text {asym }} \mathrm{CH}$ of $\left(\mathrm{C}_{19} \mathrm{H}_{20} \mathrm{H}_{21}\right)$ \\
\hline 58 & 2979 & 3109.66 & 2979.09 & 24.6775 & 2988.77 & $v_{\text {asym }} \mathrm{CH}$ of $\left(\mathrm{C}_{19} \mathrm{H}_{20} \mathrm{H}_{21}\right)$ \\
\hline 59 & 2991 & 3124.55 & 2992.59 & 1.0567 & 3003.92 & $v_{\text {asym }} \mathrm{CH}$ of $\left(\mathrm{C}_{19} \mathrm{H}_{3}\right)(81)$ \\
\hline 60 & - & 3128.17 & 2995.88 & 48.0606 & 3012.34 & $v_{\text {asym }} \mathrm{CH}$ of $\left(\mathrm{C}_{19} \mathrm{H}_{3}\right)(83)$ \\
\hline 61 & 3025 & 3167.46 & 3031.48 & 0.2119 & 3058.37 & $v(\mathrm{CH})_{\text {ring }}(93)$ \\
\hline 62 & 3060 & 3179.02 & 3041.94 & 10.4115 & 3071.30 & $\nu(\mathrm{CH})_{\text {ring }}(94)$ \\
\hline 63 & 3070 & 3189.50 & 3051.43 & 14.3460 & 3082.53 & $v(\mathrm{CH})_{\text {ring }}(95)$ \\
\hline 64 & 3090 & 3206.04 & 3066.38 & 5.7655 & 3109.14 & $v(\mathrm{CH})_{\text {ring }}(89)$ \\
\hline 65 & 3108 & 3227.43 & 3085.72 & 5.2158 & 3131.98 & $\nu(\mathrm{CH})_{\text {ring }}(85)$ \\
\hline 66 & 3458 & 3670.40 & 3482.74 & 117.92 & 3781.02 & $\nu(\mathrm{OH})(85)$ \\
\hline
\end{tabular}

${ }^{a}$ Assignments and potential energy distribution (PED) (contributing > 10\%) for vibrational normal mode. Types of vibration: $v$ : stretching; $\delta$ : deformation; o: out-of-plane bending; $\beta$ : in-plane bending; $\rho$ : rocking; $\tau$ : torsion; sym: symmetric; asym: asymmetric.

${ }^{\mathrm{b}}$ Normal mode number.

thereby they are generally mixed with other $\mathrm{CO}$ and $\mathrm{CH}_{3}$ torsions.

6.3. The $C=O$ Vibrations. The $\mathrm{C}=\mathrm{O}$ stretch of carboxylic acids is identical to the $\mathrm{C}=\mathrm{O}$ stretch in ketones, which is expected in the region 1740-1660 $\mathrm{cm}^{-1}$ [28]. Krishnakumar et al. [29] observed very strong band at $1661 \mathrm{~cm}^{-1}$ in the IR spectrum for 1-naphthyl acetic acid, and it is assigned to the $\mathrm{C}=\mathrm{O}$ stretching vibrations. In the present work a strong band observed in the FTIR spectrum at $1683 \mathrm{~cm}^{-1}$ is assigned to the $\mathrm{C}=\mathrm{O}$ stretching vibrations. The calculated peak is at $1677 \mathrm{~cm}^{-1}$ with $\mathrm{PED}$ value of $82 \%$ as reported in Table 2 .

6.4. The $\mathrm{COH}$ Vibrations. The $\mathrm{CO}$ stretching vibration is assigned to the band at $959 \mathrm{~cm}^{-1}$ in the FTIR spectrum. The predicted frequency shows a good correlation. The PED value is $83 \%$ for mode \#27 and $22 \%$ for mode \#28; most of the CO vibrations are mixed vibrations as shown in Table 2.
The $\mathrm{OH}$ group gives rise to three vibrations, namely, stretching, in-plane bending, and out-of-plane bending. The $\mathrm{OH}$ group vibrations are likely to be the most sensitive to the environment, so they show pronounced shifts in the spectra of the hydrogen-bonded species. In the case of unsubstituted phenol it has been shown that the frequency of $\mathrm{OH}$ stretching vibration in the gas phase is $3657 \mathrm{~cm}^{-1}$ [27]. Subramanian et al. [30] report the infrared spectrum of 2,5-di-tert-butylhydroquinone and assign the strong band at $3402 \mathrm{~cm}^{-1}$ to the $\mathrm{OH}$ stretching vibration. Koczon et al. [22] studied the experimental and theoretical IR and Raman spectra of picolinic, nicotinic, and isonicotinic acids and assigned the bands at 3437,3447 , and $3436 \mathrm{~cm}^{-1}$ for these molecules to the $\mathrm{OH}$ stretching vibration. In the FTIR spectrum of the title compound, the band observed at $3458 \mathrm{~cm}^{-1}$ is assigned to the $\mathrm{OH}$ stretching mode. The computed wavenumber is at $3482 \mathrm{~cm}^{-1}$ with a PED value of $85 \%$. The carboxylic acids show in-plane bending band of $\mathrm{OH}$ at $1134 \mathrm{~cm}^{-1}$ (FTIR). This band is well reproduced 
by the calculated and scaled one at $1151 \mathrm{~cm}^{-1}$. The PED calculation predicts the existence of another $\mathrm{OH}$ in-plane bending at $1365 \mathrm{~cm}^{-1}$. This band is well correlated with the one at $1363 \mathrm{~cm}^{-1}$ in the experimental infrared spectrum. The calculated frequencies at $416,418,445,506$, and $807 \mathrm{~cm}^{-1}$ are assigned to the $\mathrm{OH}$ out-of-plane bending. The last band is well correlated with the experimental one at $796 \mathrm{~cm}^{-1}$. These normal modes are mixed with the skeleton bending vibrations and torsions. The predicted PEDs are about $50 \%$.

6.5. The Methyl Group Vibrations. For the assignment of $\mathrm{CH}_{3}$ group frequencies, nine fundamentals can be associated to each $\mathrm{CH}_{3}$ group [31-33]. The $\mathrm{CH}$ stretching in $\mathrm{CH}_{3}$ occurs at lower frequencies than those of aromatic ring $\left(3100,3000 \mathrm{~cm}^{-1}\right)$. Moreover, the asymmetric stretch is usually at higher wavenumber than the symmetric stretch. In the present work, in the experimental spectrum the $\mathrm{CH}_{3}$ asymmetric stretching frequency is assigned to the bands at 2991 and $2979 \mathrm{~cm}^{-1}$, whereas the symmetric stretching frequency is assigned to the band at $2935 \mathrm{~cm}^{-1}$. These bands are well reproduced by the calculated ones at 2995, 2992, 2979 , and $2977 \mathrm{~cm}^{-1}$ for the asymmetric stretching and 2920 , $2917 \mathrm{~cm}^{-1}$ for the symmetric stretching vibrations. The PED values for these normal modes are greater than $70 \%$.

For methyl substituted benzene derivatives, the antisymmetric and symmetric deformation vibrations of methyl group normally appear in the region $1465-1440 \mathrm{~cm}^{-1}$ and $1390-1370 \mathrm{~cm}^{-1}$, respectively $[21,31,34]$. We attributed the observed doublet in the FTIR spectrum at 1465 and $1457 \mathrm{~cm}^{-1}$ to the asymmetric deformation of $\mathrm{CH}_{3}$ vibrations. These bands are well correlated with those calculated at 1480, 1473, and $1462 \mathrm{~cm}^{-1}$ with PED values greater than $80 \%$. The bands at 1374 and $1387 \mathrm{~cm}^{-1}$ (FTIR) are assigned to the symmetric $\mathrm{CH}_{3}$ deformation. The simulated frequencies at 1397 and $1377 \mathrm{~cm}^{-1}$ represent good correlations for the symmetric $\mathrm{CH}_{3}$ deformations. The normal modes no. $28, \# 30$, \#33, \#38, and \#41 are assigned to the $\mathrm{CH}_{3}$ rocking vibrations. These vibrations find location with the $\mathrm{CH}$ ring bending vibrations in the range from 984 to $1280 \mathrm{~cm}^{-1}$. This is in concordance with Subramanian et al. [30] assignments of the infrared bands of 2, 5-di-tert-butyl-hydroquinone. They reported that the rocking vibrations of methyl group for this molecule take location in the range from 837 to $1247 \mathrm{~cm}^{-1}$, and they are mixed with the $\mathrm{CH}$ ring vibrations.

\section{HOMO-LUMO Analysis}

The total energy, energy gap, and dipole moment have an effect on the stability of a molecule. In this work we calculate these parameters for title compound in gas phase and solvent. For this purpose we used B3LYP/6-311+G $\mathrm{G}^{* *}$ level of theory. Obtained results are reported in Table 3.

The energy gap between the HOMO and LUMO is an important parameter in determining molecular electrical transport proprieties and molecular stability. The energy gap between the HOMO and the LUMO is a critical parameter in determining the molecular electrical transport proprieties
TABLE 3: Calculated energies of 2-hydroxy-2-methyl-1-phenylpropan-1-one in gas, DMSO, and chloroform.

\begin{tabular}{lccc}
\hline DFT/B3LYP/6-311+G & Gas & DMSO & Chloroform \\
\hline$E_{\text {total }}$ (hartree) & -538.8921 & -538.9054 & -538.9013 \\
$E_{\text {HOMO }}(\mathrm{eV})$ & -7.456 & -7.374 & -7.401 \\
$E_{\text {LUMO }}(\mathrm{eV})$ & -2.231 & -2.204 & -2.258 \\
$\Delta E_{\text {HOMO-LUMO gap }}(\mathrm{eV})$ & 5.225 & 5.17 & 5.143 \\
$E_{\text {HOMO-1 }}(\mathrm{eV})$ & -7.592 & -7.483 & -7.510 \\
$E_{\text {LUMO+1 }}(\mathrm{eV})$ & -0.925 & -0.707 & -0.734 \\
$\Delta E_{\text {HOMO-1-LUMO+1 gap }}(\mathrm{eV})$ & 6.667 & 6.776 & 6.776 \\
\hline Dipole moment (Debye) & 4.1536 & 5.6353 & 5.1924 \\
\hline
\end{tabular}

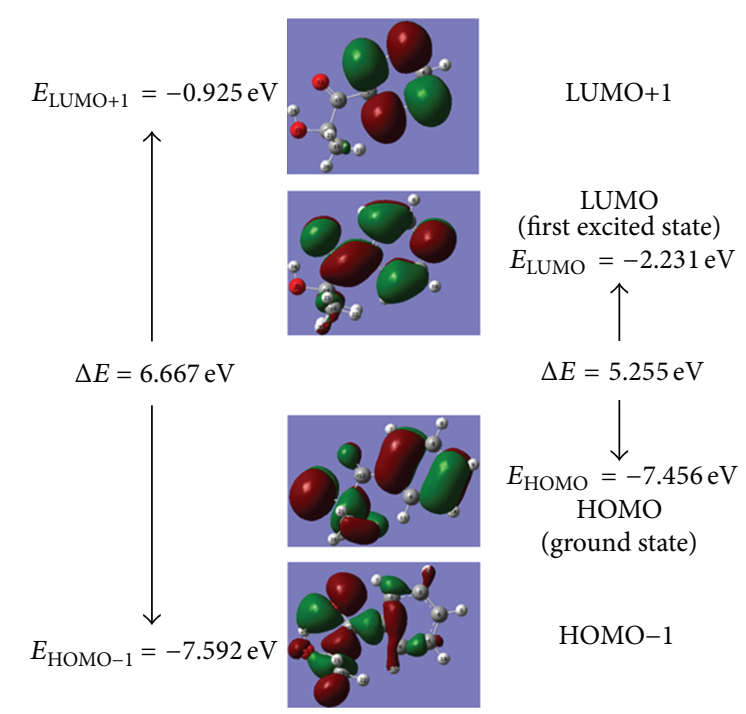

FIGURE 6: Molecular orbital frontier of 2-hydroxy-2-methyl-1phenylpropan-1-one: representation of the HOMO, HOMO-1, LUMO, and LUMO+1 orbitals.

because it is a measure of electron conductivity. The energy values of HOMO are computed $-7.456,-7.374$, and $-7.401 \mathrm{eV}$ and LUMO are $-2.231,-2.204$, and $-2.258 \mathrm{eV}$, and the energy gap values are 5.225, 5.17, and $5.143 \mathrm{eV}$ in gas phase, DMSO and chlorophorm for 2-hydroxy-2-methyl-1-phenylpropan1-one molecule, respectively. Lower value of the energy gap explains the eventual charge transfer interactions taking place within the molecule. In order to understand the bonding scheme of the title molecule we draw the surfaces of the orbitals frontier. We examine the four important molecular orbitals: the highest and the second highest occupied MOs and the lowest and the second lowest unoccupied MOs. These molecular orbitals are represented in Figure 6.

According to Figure 6 the charge distribution for the HOMO is localized on the $\mathrm{C}=\mathrm{C}$ ring, $\mathrm{OH}$, and $\mathrm{CH}$ of methyl group. The LUMO is characterized by a charge distribution at $\mathrm{CH}$ bond on the ring and $\mathrm{C}_{1} \mathrm{C}_{12}$ bond.

\section{Conclusions}

A meticulous conformational search of the title molecule leads to a unique stable conformation. For this purpose 
the calculations were conducted with reliable DFT and HF levels of theory using $6-311+G^{* *}$ basis set. The fully optimized geometries at HF and DFT (B3LYP) were compared with the experimental data of related compounds presented in the literature. The comparison shows a good agreement. Infrared spectra were recorded, and the vibrational bands were assigned on the basis of the PED calculations obtained from B3LYP/6-311+ $\mathrm{G}^{* *}$ calculations. A very good agreement between the observed and the calculated wavenumbers was observed. The scaled frequencies at $\mathrm{HF} / 6-311+\mathrm{G}^{* *}$ level of theory are in general greater than those calculated at B3LYP/6-311+G**. The latter ones are more accurate. Through the molecular orbital analysis we found that the energy gap between HOMO and LUMO is lower; this would explain the eventual charge transfer in the title molecule.

\section{References}

[1] N. S. Allen, "Photoinitiators for UV and visible curing of coatings: mechanisms and properties," Journal of Photochemistry and Photobiology A, vol. 100, no. 1-3, pp. 101-107, 1996.

[2] J. P. Fouassier, Photoinitiation, Photopolymerization and Photocuring, Hanser Publishers, Munich, Germany, 1995.

[3] B. Seidl, R. Liska, and G. Grabner, "New photocleavable structures III: photochemistry and photophysics of pyridinoyl and benzoyl-based photoinitiators," Journal of Photochemistry and Photobiology A, vol. 180, no. 1-2, pp. 109-117, 2006.

[4] H. Kaczmarek and I. Vukovic-Kwiatkowska, "Preparation and characterization of interpenetrating networks based on polyacrylates and poly(lactic acid)," eXPRESS Polymer Letters, vol. 6, no. 1, pp. 78-94, 2012.

[5] G. A. Sim, J. M. Robertson, and T. H. Goodwin, "The crystal and molecular structure of benzoic acid," Acta Crystallographica, vol. 8, pp. 157-164, 1955.

[6] F. J. Strieter and D. H. Templeton, "Crystal structure of butyric acid," Acta Crystallographica, vol. 15, pp. 1240-1244, 1962.

[7] P. S. Binil, Y. S. Mary, H. T. Varghese, C. Yohannan Panicker, M. R. Anoop, and T. K. Manujkumar, "Infrared and Raman spectroscopic analyses and theoretical computation of 4butyl-1-(4-hydroxyphenyl)-2-phenyl-3,5-pyrazolidinedione," Spectrochimica Acta Part A, vol. 94, pp. 101-109, 2012.

[8] L. Lecamp, B. Youssef, C. Bunel, and P. Lebaudy, "Photoinitiated polymerization of a dimethacrylate oligomer-part 2: kinetic studies," Polymer, vol. 40, no. 6, pp. 1403-1409, 1999.

[9] F. Burel, L. Lecamp, B. Youssef, C. Bunel, and J. M. Saiter, "Synthesis and photoinitiated polymerization of a new urethane acrylate monomer: influence of polymerization temperature," Thermochimica Acta, vol. 326, no. 1-2, pp. 133-141, 1999.

[10] L. Lecamp, B. Youssef, C. Bunel, and P. Lebaudy, "Photoinitiatedpolymerization of a dimethacrylate oligomer-part 3: postpolymerization study," Polymer, vol. 40 , no. 23 , pp. 63136320, 1999.

[11] H. Xu, F. Qiu, Y. Wang, W. Wu, D. Yang, and Q. Guo, "UV-curable waterborne polyurethane-acrylate: preparation, characterization and properties," Progress in Organic Coatings, vol. 73, no. 1, pp. 47-53, 2012.

[12] L. Lecamp, B. Youssef, C. Bunel, and P. Lebaudy, "Photoinitiated polymerization of a dimethacrylate oligomer: 1. Influence of photoinitiator concentration, temperature and light intensity," Polymer, vol. 38, no. 25, pp. 6089-6096, 1997.
[13] A. D. Becke, "Density-functional thermochemistry. III. The role of exact exchange," The Journal of Chemical Physics, vol. 98, no. 7, pp. 5648-5652, 1993.

[14] C. Lee, W. Yang, and R. G. Parr, "Development of the ColleSalvetti correlation-energy formula into a functional of the electron density," Physical Review B, vol. 37, no. 2, pp. 785-789, 1988.

[15] V. K. Rastogi, M. A. Palafox, R. P. Tanwar, and L. Mittal, “3,5difluorobenzonitrile: Ab initio calculations, FTIR and Raman spectra," Spectrochimica Acta Part A, vol. 58, no. 9, pp. 19872004, 2002.

[16] H. Yoshida, A. Ehara, and H. Matssura, "Density functional vibrational analysis using wavenumber-linear scale factors," Chemical Physics Letters, vol. 325, no. 4, pp. 477-483, 2000.

[17] J. Baker, A. A. Jarzecki, and P. Pulay, "Direct scaling of primitive valence force constants: an alternative approach to scaled quantum mechanical force fields," Journal of Physical Chemistry A, vol. 102, no. 8, pp. 1412-1424, 1998.

[18] M. J. Frisch, G. W. Trucks, H. B. Schlegel et al., Gaussian 03, Revision B.01, Gaussian, Inc., Pittsburgh, Pa, USA, 2003.

[19] J. M. L. Martin and C. van Alsenoy, GAR2PED: A Program to obtain a Potential Energy Distribution from a Gaussian Archive Record, University of Antwerp, Antwerp, Belgium, 2009.

[20] P. Pulay, G. Fogarasi, F. Pang, and J. E. Boggs, "Systematic $\mathrm{ab}$ initio gradient calculation of molecular geometries, force constants, and dipole moment derivatives," Journal of the American Chemical Society, vol. 101, no. 10, pp. 2550-2560, 1979.

[21] M. Silverstein, G. C. Basseler, and C. Morill, Spectrometric Identification of Organic Compounds, Wiley, New York, NY, USA, 1981.

[22] P. Koczon, J. Cz. Dobrowolski, W. Lewandowski, and A. P. Mazurek, "Experimental and theoretical IR and Raman spectra of picolinic, nicotinic and isonicotinic acids," Journal of Molecular Structure, vol. 655, no. 1, pp. 89-95, 2003.

[23] M. Karabacak, Z. Cinar, M. Kurt, S. Sudha, and N. Sundaraganesan, "FT-IR, FT-Raman, NMR and UV-vis spectra, vibrational assignments and DFT calculations of 4-butyl benzoic acid," Spectrochimica Acta Part A, vol. 85, no. 1, pp. 179-189, 2012.

[24] P. B. Nagabalasubramanian, M. Karabacak, and S. Periandy, "FT-IR, FT-Raman, ab initio and DFT structural, vibrational frequency and HOMO-LUMO analysis of 1-naphthaleneacetic acid methyl ester," Spectrochimica Acta Part A, vol. 82, no. 1, pp. 169-180, 2011.

[25] N. B. Colthup, L. H. Daly, and S. E. Wiberley, Introduction to Infrared and Raman Spectroscopy, Academic Press, New York, NY, USA, 1964.

[26] L. J. Bellamy, The Infrared Spectra of Complex Molecules, Chapman and Hall, 1980.

[27] G. Varsanyi, Assignments for Vibrational Spectra of Seven Hundred Benzene Derivatives, vol. 1-2, Adam Hilger, 1974.

[28] D. L. Vein, N. B. Colthup, W. G. Fateley, and J. G. Grasselli, The Handbook of Infrared and Raman Characteristic Frequencies of Organic Molecules, Academic Press, San Diego, Calif, USA, 1991.

[29] V. Krishnakumar, R. Mathammal, and S. Muthunatesan, "FT-IR and Raman spectra vibrational assignments and density functional calculations of 1-naphthyl acetic acid," Spectrochimica Acta Part A, vol. 70, no. 1, pp. 210-216, 2008.

[30] N. Subramanian, N. Sundaraganesan, O. Dereli, and E. Turkkan, "FT-IR, FT-Raman spectra, density functional computations of the vibrational spectra and molecular conformational analysis of 2,5-di-tert-butyl-hydroquinone," Spectrochimica Acta Part A, vol. 83, no. 1, pp. 165-174, 2011. 
[31] E. B. Wilson, J. C. Decius, and P. C. Cross, Molecular Vibrations: The Theory of Infrared and Raman Vibrational Spectra, McGrawHill Book Company, New York, NY, USA, 1955.

[32] O. Dereli, Y. Erdogdu, M. T. Gulluogu, E. Turkkan, A. Ozmen, and N. Sundaraganesan, "Vibrational spectral and quantum chemical investigations of tert-butyl-hydroquinone," Journal of Molecular Structure, vol. 1012, pp. 168-176, 2012.

[33] P. Chinna Babu, N. Sundaraganesan, O. Dereli, and E. Türkkan, "FT-IR, FT-Raman spectra, density functional computations of the vibrational spectra and molecular geometry of butylated hydroxy toluene," Spectrochimica Acta Part A, vol. 79, no. 3, pp. 562-569, 2011.

[34] S. Kalaichelvan, N. Sundaraganesan, O. Dereli, and U. Saying, "Experimental, theoretical calculations of the vibrational spectra and conformational analysis of 2,4-di-tert-butylphenol," Spectrochimica Acta Part A, vol. 85, no. 1, pp. 198-209, 2012. 

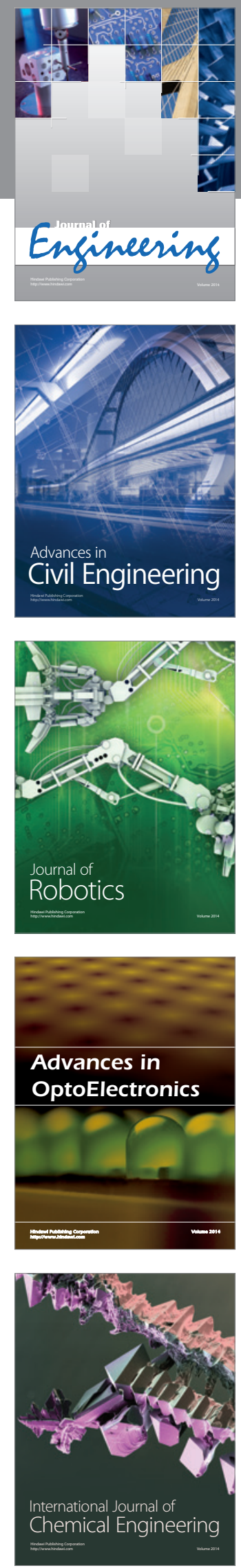

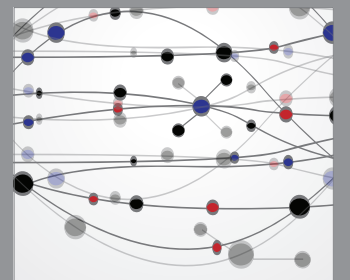

The Scientific World Journal
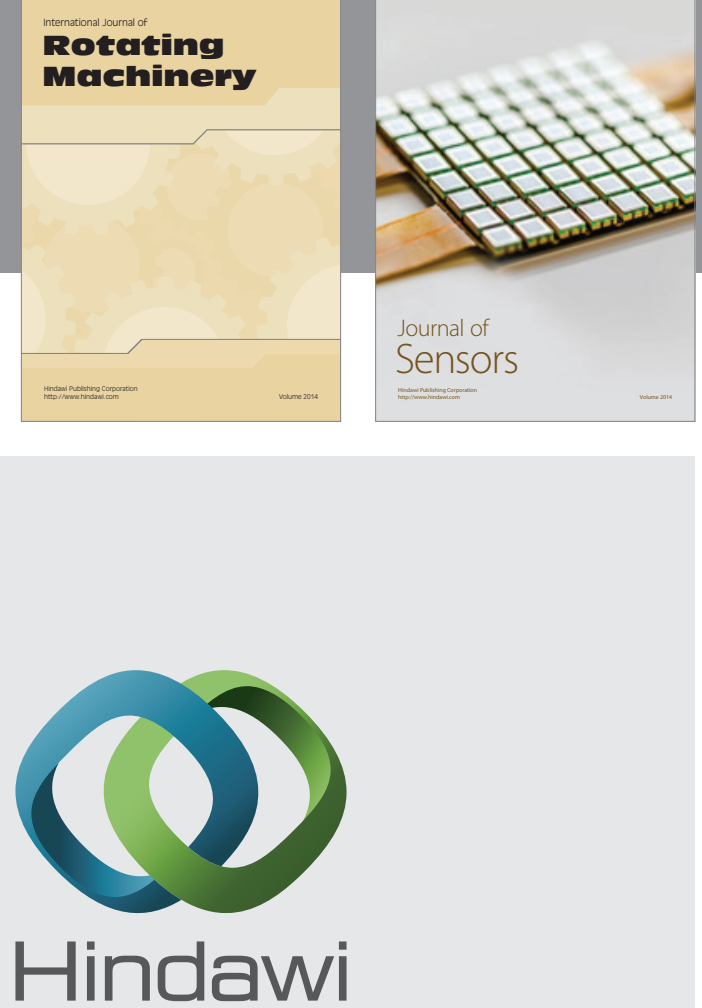

Submit your manuscripts at http://www.hindawi.com
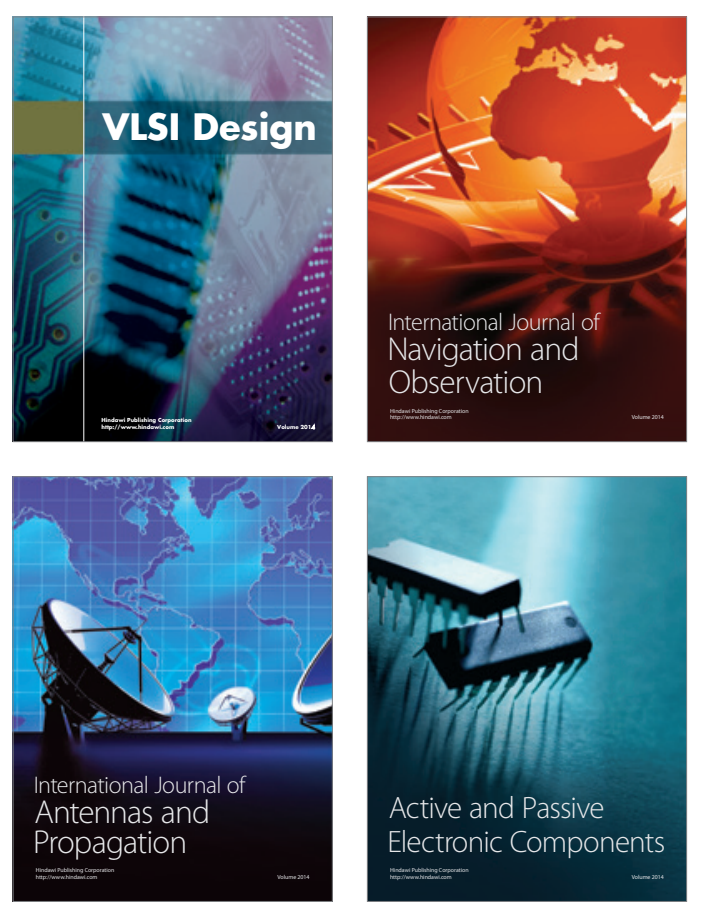
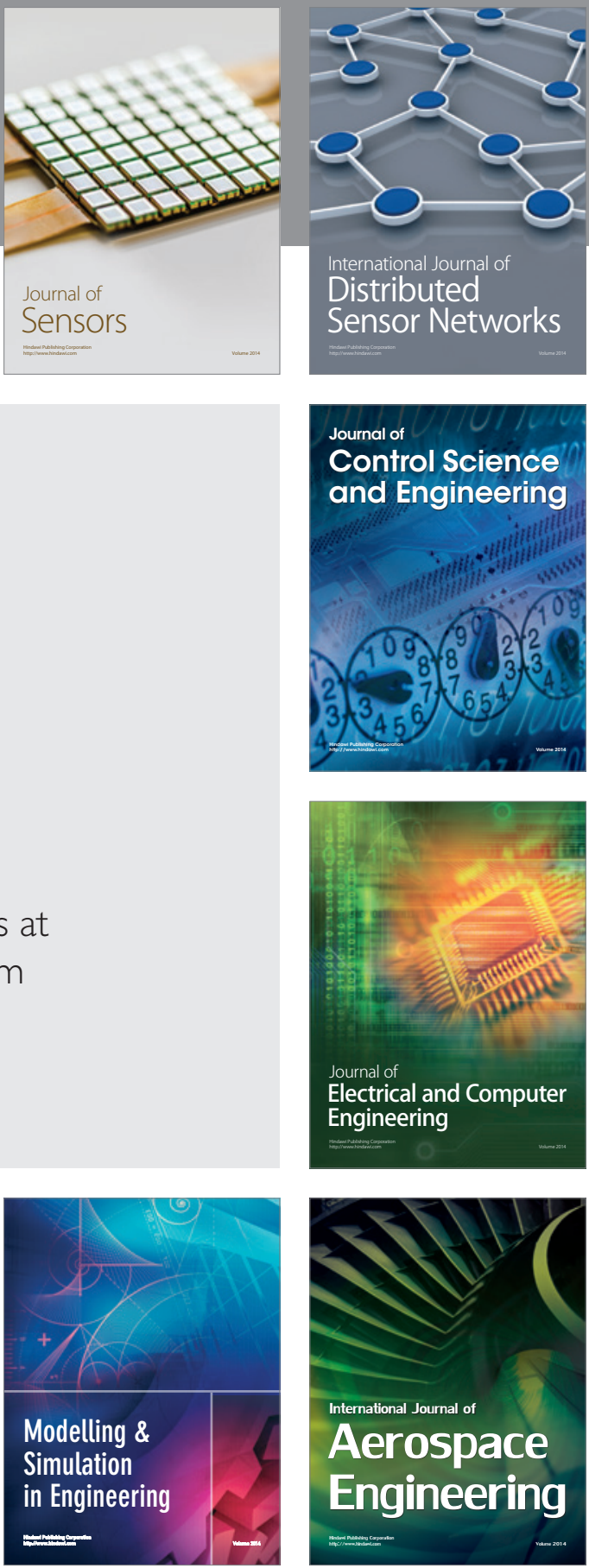

Journal of

Control Science

and Engineering
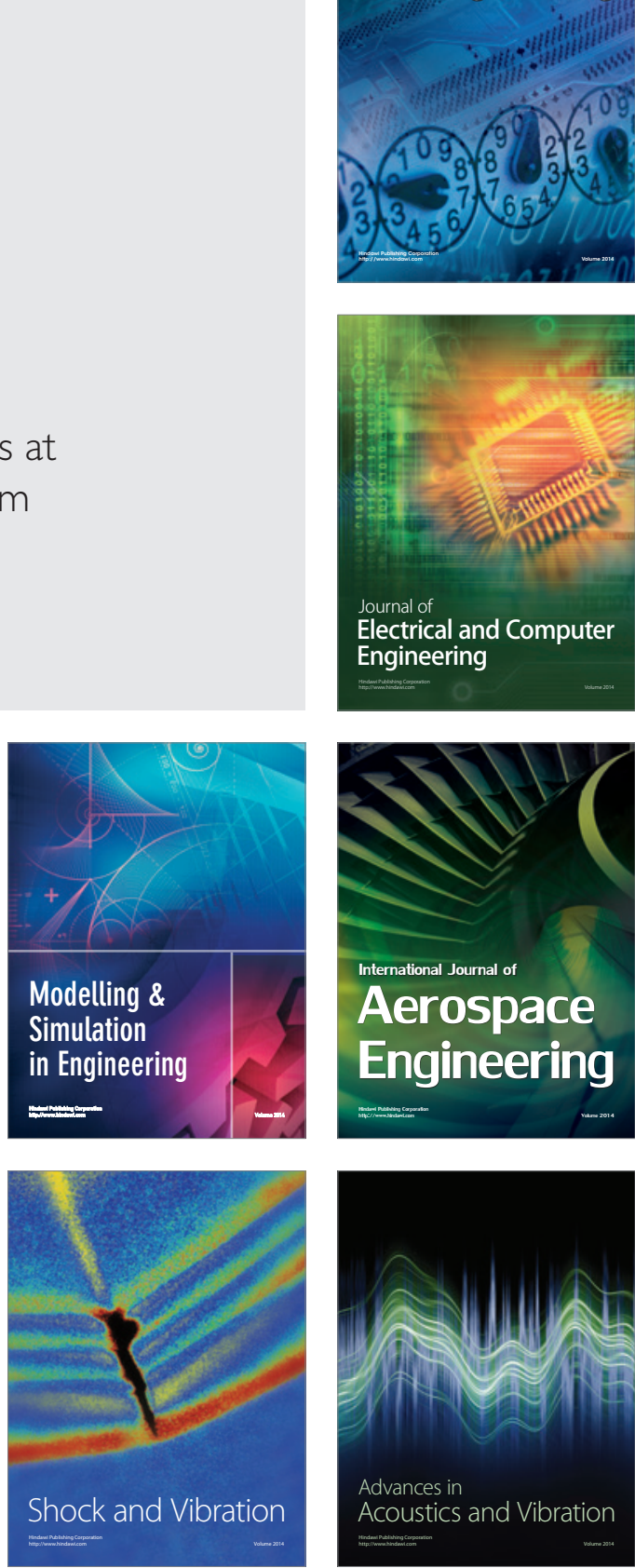